Audiology

Neurotology
Audiol Neurotol 2017;22:282-291

DOI: $10.1159 / 000485022$
Received: April 20, 2017

Accepted: November 2, 2017

Published online: January 12, 2018

\title{
Toward Optimizing Vestibular Evoked Myogenic Potentials: Normalization Reduces the Need for Strong Neck Muscle Contraction
}

\author{
Kimberley S. Noij ${ }^{a}$ Barbara S. Herrmann ${ }^{\text {b, d }}$ Steven D. Rauch ${ }^{\text {a,d }}$ \\ John J. Guinan Jr. ${ }^{c, d}$ \\ Departments of a Otolaryngology and ${ }^{\mathrm{b}}$ Audiology and ${ }^{\mathrm{c}}$ Eaton Peabody Lab, Massachusetts Eye and Ear Infirmary, \\ and ${ }^{\mathrm{d}}$ Department of Otology and Laryngology, Harvard Medical School, Boston, MA, USA
}

\section{Keywords}

Cervical vestibular evoked myogenic potential · Muscle contraction · Normalization

\begin{abstract}
Background: The cervical vestibular evoked myogenic potential (cVEMP) represents an inhibitory reflex of the saccule measured in the ipsilateral sternocleidomastoid muscle (SCM) in response to acoustic or vibrational stimulation. Since the CVEMP is a modulation of SCM electromyographic (EMG) activity, cVEMP amplitude is proportional to muscle EMG amplitude. We sought to evaluate muscle contraction influences on CVEMP peak-to-peak amplitudes (VEMPpp), normalized cVEMP amplitudes (VEMPn), and inhibition depth (VEMPid). Methods: cVEMPs at $500 \mathrm{~Hz}$ were measured in 25 healthy subjects for 3 SCM EMG contraction ranges: 45-65, 65-105, and 105-500 $\mu \mathrm{V}$ root mean square (r.m.s.). For each range, we measured cVEMP sound level functions (93-123 dB peSPL) and sound off, meaning that muscle contraction was measured without acoustic stimulation. The effect of muscle contraction amplitude on VEMPpp, VEMPn, and VEMPid and the ability to distinguish cVEMP presence/absence were evaluated. Results: VEMPpp amplitudes were significantly greater at higher muscle contractions. In contrast, VEMPn and VEMPid showed no significant effect of muscle contraction.
\end{abstract}

Cohen's $d$ indicated that for all 3 cVEMP metrics contraction amplitude variations produced little change in the ability to distinguish CVEMP presence/absence. VEMPid more clearly indicated saccular output because when no acoustic stimulus was presented the saccular inhibition estimated by VEMPid was zero, unlike those by VEMPpp and VEMPn. Conclusion: Muscle contraction amplitude strongly affects VEMPpp amplitude, but contractions 45-300 $\mu \mathrm{V}$ r.m.s. produce stable VEMPn and VEMPid values. Clinically, there may be no need for subjects to exert high contraction effort. This is especially beneficial in patients for whom maintaining high SCM contraction amplitudes is challenging.

ㄷ) 2018 S. Karger AG, Basel

\section{Introduction}

Cervical vestibular evoked myogenic potentials (cVEMPs) are responses to acoustic or mechanical stimuli measured with electromyography (EMG) on the sternocleidomastoid muscle (SCM). The ipsilateral inhibition produced by this vestibulocollic reflex is measured

The material of this manuscript has been accepted for poster presentation by the Association of Research in Otolaryngology for the Midwinter meeting in 2017.

\section{KARGER}

(c) 2018 S. Karger AG, Basel

E-Mail karger@karger.com

www.karger.com/aud
John J. Guinan Jr., $\mathrm{PhD}$

243 Charles Street

Boston, MA 02114 (USA)

E-Mail John_Guinan@meei.harvard.edu 
with surface electrodes on the tonically contracted SCM [Colebatch et al., 1994]. This SCM response is predominantly of saccular origin and provides a measure of the integrity of the saccule and inferior vestibular nerve [Curthoys, 2010]. Typically a cVEMP consists of 2 peaks: a positive peak and a negative peak, referred to as $\mathrm{P} 1$ and $\mathrm{N} 1$, occurring approximately 13 and $23 \mathrm{~ms}$ after sound onset for clicks [Colebatch et al., 1994].

Several metrics can be used to analyze cVEMPs. A common metric is the peak-to-peak amplitude difference between P1 and N1 (VEMPpp). VEMPpp increases with increased muscle contraction [Akin and Murnane, 2001; Akin et al., 2004; Bogle et al., 2013; Colebatch et al., 1994; Davenport, 2010; Isaacson et al., 2006; Lim et al., 1995; McCaslin et al., 2014; Ochi et al., 2001; Rosengren, 2015]. To make VEMPpp comparisons across subjects, contraction amplitude can be kept the same across subjects. Alternately, one can correct for differences in contraction amplitude by dividing VEMPpp by EMG amplitude which yields the normalized VEMPpp (VEMPn) [Isaacson et al., 2006; McCaslin et al., 2014; Rosengren, 2015; van Tilburg et al., 2014]. A third metric, VEMP inhibition depth (VEMPid), estimates the "inhibition depth," defined as the fractional inhibition of SCM motoneuron response produced by activation of the saccule [Prakash et al., 2015]. VEMPid uses a template correlation method that resembles a matched filter for the detection of a signal (the cVEMP) in noise. VEMPid provides normalization and improved sensitivity at low response levels [Prakash et al., 2015].

Our focus in this study is to determine the muscle contraction amplitude range over which normalization works well. Normalization produces a constant VEMPn value when VEMPpp grows linearly with contraction amplitude as measured by the muscle EMG amplitude. Many studies have reported that for most subjects VEMPpp increases linearly with contraction amplitude [Akin and Murnane, 2001; Akin et al., 2004; Colebatch et al., 1994; Isaacson et al., 2006; Lim et al., 1995; Ochi et al., 2001]. However, a few studies reported that for some subjects VEMPpp saturates for strong muscle contractions [Bogle et al., 2013; McCaslin et al., 2014; Rosengren, 2015]. To achieve a wide range of contraction amplitudes, several different muscle maneuvers (e.g., lifting vs. turning the head) have been used [Bogle et al., 2013; Davenport, 2010; Rosengren, 2015; Wang and Young, 2006]. However, with different maneuvers different muscles are activated which may change the pattern of activation within the SCM. Furthermore, the orientation of the saccule relative to gravity changes with dif- ferent maneuvers. For instance, Rosengren [2015] found VEMPpp versus EMG nonlinearity for low-amplitude contractions (averaged-full-wave-rectified (RECT) EMGs less than $50 \mu \mathrm{V}$ ) and excluded these from further analysis. However, this nonlinearity may be caused by the use of different head position maneuvers to produce low-amplitude EMGs. Another factor is the outcome measure considered when choosing a suitable EMG contraction range. Studies optimized for measuring cVEMP interaural asymmetry ratios (IARs) may lead to recommending stronger muscle contractions [Rosengren, 2015]. However, some patients with nominally unilateral Ménière disease show abnormal cVEMPs in their "unaffected" ear, which indicates that the IAR is a flawed VEMP metric [Rauch et al., 2004]. Here we do not consider the IAR and only consider optimizing individual normalized cVEMP measurements.

When obtaining cVEMPs in the clinic, a single means of achieving SCM contraction is used, and the question is: What is the range of SCM contraction amplitudes that provides consistent cVEMP outcomes? For cVEMP measurements, patients in our clinic are now encouraged to produce contractions that yield root-mean-square (r.m.s.) EMGs $>65 \mu \mathrm{V}$ (approx. $50 \mu \mathrm{V}$ RECT) by sitting up straight and turning their head away from the stimulated ear. Many, especially older, patients find it challenging to reach this contraction amplitude. To avoid requiring patients to make contractions that are unnecessarily strong, and to reduce discomfort, it is important to know how much contraction amplitude influences cVEMP metrics, especially for low-amplitude contractions.

We collected cVEMP data from healthy subjects using 1 head-turning maneuver to achieve specified low-amplitude muscle contraction ranges. Our primary aims were: (1) to determine how much muscle contraction amplitude influences cVEMP metrics, (2) to evaluate across sound intensities how well VEMPn and VEMPid correct for differences in contraction amplitudes, and (3) to determine the preferred target contraction range. We found that contractions with $45-500 \mu \mathrm{V}$ r.m.s. EMGs yielded relatively uniform VEMPn and VEMPid values.

\section{Methods}

\section{Subjects}

Twenty-five healthy subjects were included: 9 male and 16 female (mean age: 42.84 years, range: $22-76$ years). All subjects received air and bone conduction audiograms at 250, 500, 750, 1,000, 2,000 , and $4,000 \mathrm{~Hz}$. Subjects were excluded if they had an air-bone gap $>10 \mathrm{~dB}$ or a history of balance issues, hearing loss or neurological disease. Informed consent was obtained and signed by all 
subjects. This study was approved by the Human Studies Committee of the Massachusetts Eye and Ear Infirmary (No. 13-097H, Principal investigator: S.D. Rauch).

\section{cVEMP Testing}

To contract the SCM, subjects sat up straight and turned their head away from the stimulated ear. EMG activity was recorded from 4 surface electrodes: a positive electrode on the middle belly of each SCM, a reference electrode at the midline point between the SCM attachments to the sternum, and a ground electrode on the midline forehead. Ipsilateral SCM EMG was monitored while subjects maintained rms EMGs within 1 of 3 ranges (45-65, 65105 , and $105-500 \mu \mathrm{V}$ r.m.s.) as requested by the experimenter. In our data, r.m.s. EMG values averaged 1.32 (1.27-1.45 across subjects) times higher than full-wave rectified EMG values; thus, the range divisions are: $34.1-49.2,49.2-79.5$, and $79.5 \mu \mathrm{V}$ to 378.8 RECT. Before the first test, subjects briefly practiced maintaining SCM contractions within the 3 ranges. During both practice and tests, subjects received verbal feedback on their muscle contraction amplitude. EMG activity was amplified, bandpass filtered and sampled at $50 \mathrm{kHz}$ with a 16-bit analog-to-digital converter ( National Instruments), and all responses were stored.

$500-\mathrm{Hz}$ tone bursts were generated by custom-programmed evoked potential software (National Instruments 16-bit digital I/O board) using a Blackman gating function with 2 cycle $(4.0 \mathrm{~ms})$ rise and fall times and no plateau. Tone bursts were presented monaurally with supra-aural headphones (Telephonics TDH-49) at a repetition rate of 13/s. Tone bursts were presented at 93, 103, 113, and $123 \mathrm{~dB}$ peak-equivalent sound pressure level (peSPL; $93 \mathrm{~dB}$ peSPL is $60 \mathrm{~dB} \mathrm{nHL}$ ). cVEMP responses were recorded for all 3 contraction ranges for each side and for each sound level. In addition, EMG recordings were obtained without any sound stimulus for all 3 muscle contraction ranges. For this, subjects contracted their SCM as they did when acoustically stimulated, but no sound was delivered. We randomized presentation order for sound level, side and muscle contraction range. For each recording 200-300 single EMG responses were obtained and stored.

\section{Analyses}

VEMPpp was calculated by measuring the amplitude difference between $\mathrm{P} 1$ and $\mathrm{N} 1$ in the averaged cVEMP waveform. P1 was the largest positive peak in the interval 9-20 ms, and N1 was the largest negative peak after $\mathrm{P} 1$ and before $30 \mathrm{~ms}$. Time was measured relative to the peak of the sound stimulus, which gives uniform average delays across different sound frequencies [Bogle et al., 2013; van Tilburg et al., 2014]. These acceptance windows take into account the normal variation in cVEMP latencies across subjects.

VEMPn was calculated from the peak-to-peak amplitude of the averaged, normalized cVEMP waveform. To normalize waveforms, each raw EMG trace was divided by the overall, 77-ms-long, r.m.s. amplitude of the same trace (this is termed trace-by-trace normalization) [van Tilburg et al., 2014]. VEMPn was the peak-topeak value measured from this normalized VEMP waveform as described for VEMPpp.

To compute VEMPid, a template of the expected cVEMP waveform is required, and for this we used a generic template made by averaging peak-aligned cVEMP responses from 6 other normal subjects [Noij et al., 2017]. The point-by-point correlation of each individual trace with the generic template was calculated (termed the template correlation value or TCV). VEMPid is the mean of all the 200-300 TCVs divided by the standard deviation of the TCVs and multiplied by 0.2 . A more detailed description of the VEMPid calculation (using a subject-specific template) can be found in Prakash et al. [2015]. To compensate for the generic template's lack of latency information, TCVs were calculated with the generic template's delay varied to cover the interval from 9 to $20 \mathrm{~ms}$ in 0.1 ms steps, and the largest value was used for the VEMPid. The latency that yielded the largest VEMPid at the highest sound level was used for calculating VEMPids at lower sound levels.

Patients were divided into 3 age groups: (1) 22-29, (2) 30-49, and (3) 50-76 years old, $n=6,9$, and 10, respectively. For the VEMPpp, VEMPn and VEMPid measurements, we calculated means and standard errors across subjects in every age group at each sound level, side, and muscle contraction range. Full factorial analyses of variance (ANOVAs) were performed to determine the effect of muscle contraction amplitude on each of these metrics. Age, side, sound level, and muscle contraction amplitude were considered fixed factors, and the subject was considered a variable factor. Data from both ears were pooled for the figures and subsequent analysis.

To examine the degree to which VEMPpp, VEMPn, and VEMPid were influenced by muscle contraction, we calculated the etasquared effect size $\left(\eta^{2}\right)$ for each metric and sound level. The $\eta^{2}$ is a ratio of variances and gives the proportion of the variance in the cVEMP metric that can be attributed to (in this case) changes in muscle contraction amplitude.

$$
\eta^{2}=\frac{\text { SSgroup }}{\text { SStotal }}
$$

where SS is the sum of squares; SSgroup is the sum of the squared differences between the mean $(\mu)$ of every muscle tension group ( $\mu$ group) and the total mean ( $\mu$ total), multiplied by the number in the group, ngroup, i.e.

$$
\text { SSgroup }=\sum_{1}^{3} \text { ngroup } \times(\mu \text { group }-\mu \text { total })^{2} ;
$$

and SStotal is the sum of the squared differences between every individual point and the total mean, i.e.

$$
\text { SStotal }=\sum_{i=1}^{n \text { total }}(x i-\mu \text { total })^{2} .
$$

$\eta^{2}$ values of $0.01,0.06$, and 0.14 are considered small, medium, and large, respectively [Cohen, 1988].

To determine how well each cVEMP metric was able to distinguish cVEMP presence or absence, Cohen's $d$ effect sizes were calculated using the cVEMP metrics from sound-on versus no-sound EMG measurements. Cohen's $d$ is the difference between each group mean $\left(\mu_{1}\right)$ and the corresponding no-sound mean $\left(\mu_{2}\right)$, divided by the combined standard deviation of the 2 groups $\left(\sigma_{1,2}\right)$.

$$
\text { Cohen's } D=\frac{\mu 1-\mu 2}{\sigma 1,2} \text {. }
$$

As stated earlier: for each recording 200-300 single EMG responses were obtained and used to calculate VEMPpp, VEMPn, VEMPid, $\eta^{2}$ and Cohen's $d$.

Statistical analyses were performed using Matlab (version R2014a) and SPSS (version 22.0; Chicago, IL, USA). A $p$ value of $<0.05$ was considered statistically significant. All post hoc pairwise comparisons were performed using a Bonferroni adjustment for multiple comparisons. 
Table 1. The effect of muscle contraction on VEMPpp, VEMPn, and VEMPid ( $F$ values and $p$ values) for every sound level separately

\begin{tabular}{|c|c|c|c|c|c|c|}
\hline \multirow[t]{2}{*}{ Sound level } & \multicolumn{2}{|c|}{ VEMPpp } & \multicolumn{2}{|c|}{ VEMPn } & \multicolumn{2}{|c|}{ VEMPid } \\
\hline & $F$ value & $p$ value & $F$ value & $p$ value & $F$ value & $p$ value \\
\hline $93 \mathrm{~dB}$ peSPL & 8.727 & $<0.001$ & 0.035 & 0.966 & 0.225 & 0.798 \\
\hline $103 \mathrm{~dB}$ peSPL & 11.156 & $<0.001$ & 0.103 & 0.902 & 0.015 & 0.985 \\
\hline $113 \mathrm{~dB}$ peSPL & 32.005 & $<0.001$ & 0.157 & 0.885 & 0.488 & 0.614 \\
\hline $123 \mathrm{~dB}$ peSPL & 132.353 & $<0.001$ & 0.466 & 0.628 & 1.684 & 0.187 \\
\hline
\end{tabular}

VEMPpp is significantly affected by muscle contraction amplitude for all sound levels, whereas VEMPn and VEMPid are not significantly affected by muscle contraction amplitude at any sound level. $p$ values $\leq 0.05$ are significant.

\section{Results}

Typical cVEMP waveforms from the 3 muscle contraction ranges and across sound levels are shown in Figure 1. Consistently with most previous work, VEMPpp increased almost linearly with increases in muscle contraction amplitude for the 3 muscle contraction groups regardless of age group (Fig. 2a-c). On average the increases in VEMPpp with increases in muscle contraction amplitude were significantly greater at higher sound levels as shown by a significant interaction between muscle contraction amplitude and sound level (ANOVA: $F=15.410$, $p<0.001)$. Because of this significant interaction, the effect of muscle contraction amplitude on VEMPpp was calculated separately for each sound level. For all sound levels VEMPpp was significantly higher at higher muscle contraction amplitudes with age groups combined (Table 1).

Average P1 latencies decreased from 14.0 to $13.0 \mathrm{~ms}$ for the $45-$ to $65-\mu \mathrm{V}$ and $65-$ to $105-\mu \mathrm{V}$ groups, respectively (Bonferroni adjusted $p=0.012$ ), and there was no statistically significant change comparing $65-105 \mu \mathrm{V}$ and 105-500 $\mu \mathrm{V}$ (13.0-13.5 ms; Bonferroni adjusted $p=$ $0.559)$. Average N1 latencies were not statistically significantly different across muscle contraction amplitude groups (from lowest to highest contraction group: 23.2, 22.8 , and $22.7 \mathrm{~ms} ; p=0.375$ ).

In contrast to VEMPpp, both VEMPn and VEMPid showed no significant effect of the 3 muscle contraction amplitudes, controlling for possible interactions with sound level and side (ANOVA: $F=0.338, p=0.713$, and $F=0.605, p=0.546$, respectively; Figure $2 \mathrm{~d}-\mathrm{i})$. Both VEMPn and VEMPid increased as sound level was increased and there was an interaction between age and sound level $(F=15.060, p<0.001 ; F=12.797, p<0.001)$. Therefore, the effect of sound level on VEMPn and

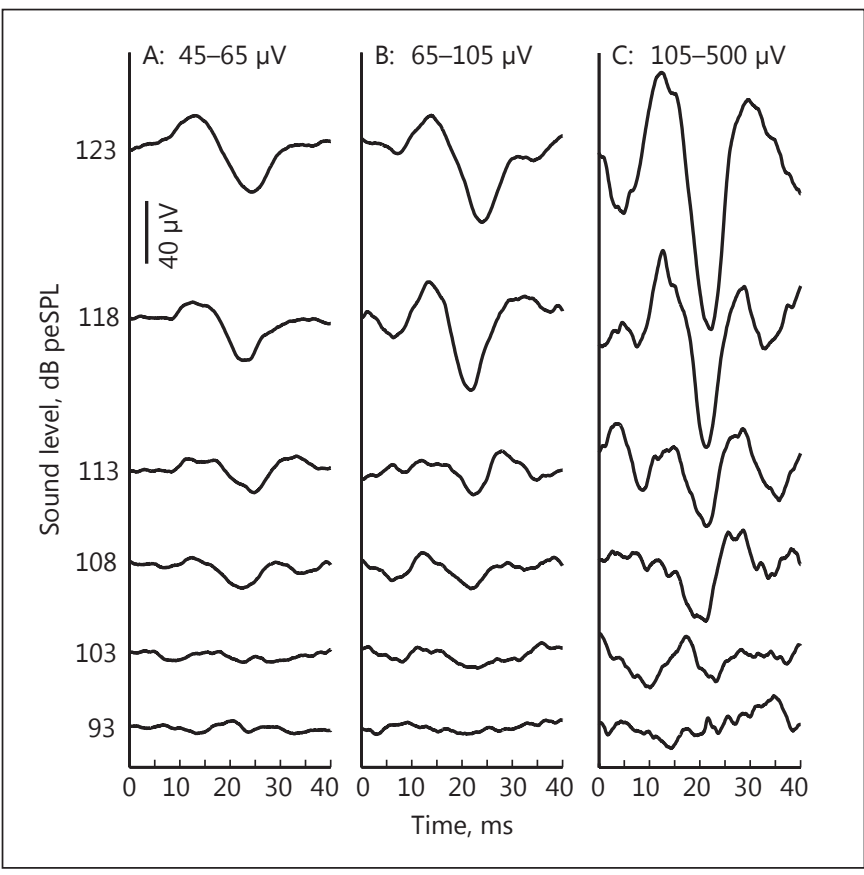

Fig. 1. Example cVEMP traces from 1 subject showing responses to different stimulus levels while contracting the SCM within the 3 different muscle contraction amplitude ranges (lanes $\mathrm{A}-\mathrm{C}$ ). Although the cVEMP amplitudes increase with contraction amplitude, the resulting larger cVEMP amplitudes do not improve the ability to determine whether a CVEMP is present or not because of the higher level residual noise. All cVEMPs displayed in this figure are averages of 200-300 single EMG responses.

VEMPid was calculated separately for each age group. The effect of sound level was significant in all age groups for both VEMPn and VEMPid (all age groups: $p<0.001$ ), and the effect of age was only significant at the highest sound levels (VEMPn at 93, 103, 113, and $123 \mathrm{~dB}$ peSPL: 


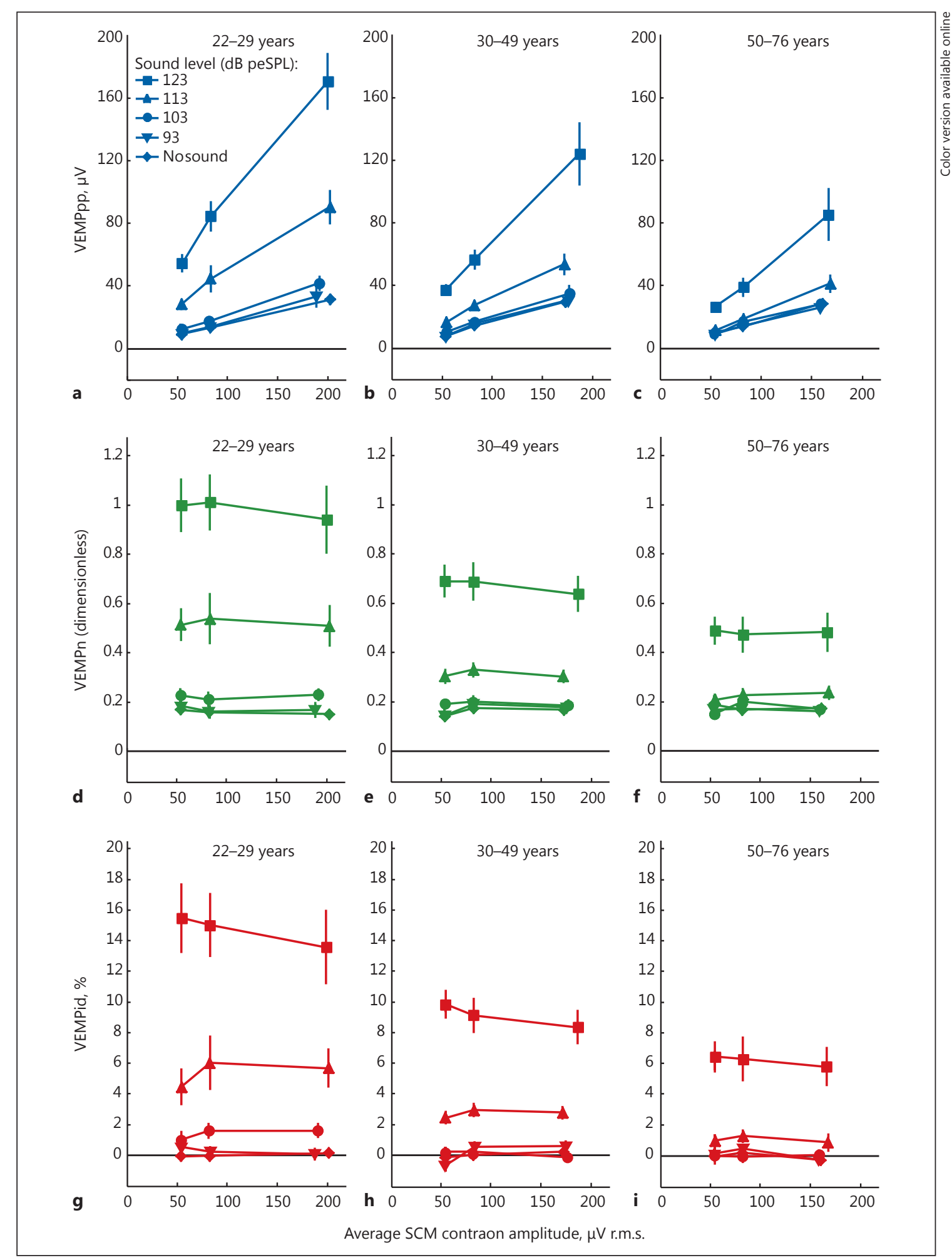

Fig. 2. Average VEMP peak-to-peak (VEMPpp) (a-c), normalized VEMP (VEMPn) (d-f), and VEMP inhibition depth (VEMPid) (gi) for the 3 age groups as functions of average muscle contraction amplitude, showing that VEMPpp grows with muscle contraction in all age groups $(\mathbf{a}-\mathbf{c})$, whereas VEMPn and VEMPid remain stable for muscle contraction amplitudes $>45 \mu \mathrm{V}$ r.m.s. in all age groups (d-i). The symbols represent data from stimulus levels 123, 113, 103, $93 \mathrm{~dB}$ peSPL, and sound off within the predefined muscle contraction ranges $(45-65,65-105$, and $105-500 \mu \mathrm{V}$ r.m.s.) from all subjects (50 ears). Error bars indicate the standard error of the mean. 


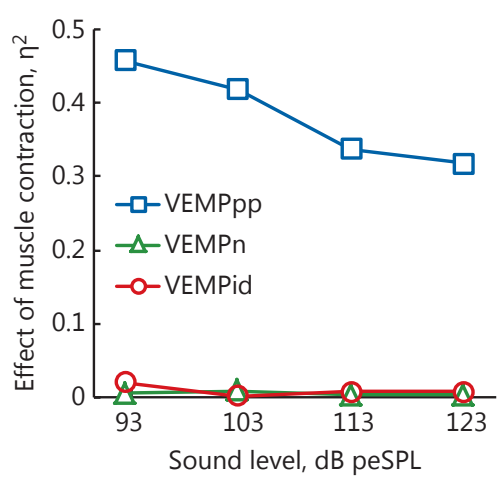

Fig. 3. $\eta^{2}$ assessment of the influence of muscle contraction amplitude on VEMPpp, VEMPn, and VEMPid. Effect sizes of 0.01, 0.06, and 0.14 are considered small, medium, and large, respectively. Muscle contraction had a large effect on VEMPpp $\left(\eta^{2}=0.32-0.45\right)$, a small effect on VEMPn $\left(\eta^{2}<0.01\right)$, and a small effect on VEMPid (0.01-0.02).

$F=0.005, p=0.995 ; F=0.650, p=0.522 ; F=25.213, p<$ $0.001 ; F=71.104, p<0.001$; VEMPid at $93,103,113$, and $123 \mathrm{~dB}$ peSPL: $F=0.038, p=0.962 ; F=2.117, p=0.121$; $F=18.085, p<0.001 ; F=68.434, p<0.001)$. In contrast to VEMPpp, the effects of muscle contraction amplitude on VEMPn and on VEMPid were not significant when each sound level was considered individually (Table 1).

To get a quantitative measure of how much muscle contraction amplitude affected the various cVEMP metrics at each sound level, we calculated the $\eta^{2}$ effect size across the 3 contraction ranges (Fig. 3). Because the effects of muscle contraction amplitude are similar amongst the different age groups, data of all age groups were combined to calculate the $\eta^{2}$ effect size. According to the standard assessments for $\eta^{2}$ (see Methods), muscle contraction amplitude had a very large effect on VEMPpp $\left(\eta^{2}=\right.$ $0.32-0.45)$, a small effect on VEMPn $\left(\eta^{2}<0.01\right)$, and a small effect on VEMPid $\left(\eta^{2}=0.01-0.02\right)$ (Fig. 3).

Since VEMPn and VEMPid did not change significantly across muscle contractions for EMGs down to at least $45 \mu \mathrm{V}$ r.m.s. (Fig. $2 \mathrm{~d}-\mathrm{i}$ ), we explored whether even lower contractions might be acceptable. Most subjects maintained their contraction amplitudes within the predefined ranges, but some occasionally produced contractions below the minimum targeted contraction of $45 \mu \mathrm{V}$ rms. We used these data when there were 100 or more responses within the 25 - to $45-\mu \mathrm{V}$ r.m.s. muscle contraction range (at $93,103,113$, and $123 \mathrm{~dB}$ peSPL in age group $1 \mathrm{n}=7,6,5,5$ ears; in age group $2 \mathrm{n}=13,11,11,13$ ears; in age group $3 n=13,13,9,11$ ears, respectively). No subject had 100 or more responses available within the 0 - to $25-\mu \mathrm{V}$ r.m.s. contraction range. In the $25-45 \mu \mathrm{V}$ rms range, VEMPpp continued its near-linear relationship with muscle contraction amplitude in all age groups, while VEMPn and VEMPid values in the 25- to $45-\mu \mathrm{V}$ r.m.s. range decreased somewhat (data not shown). None of the VEMPn or VEMPid changes in the 25- to $45-\mu \mathrm{V}$ r.m.s. range (relative to the $45-$ to $65-\mu \mathrm{V}$ r.m.s. range) were statistically significant.

The data of Figure 2 are illustrated in Figure 4 as functions of sound level. This figure shows that VEMPid values are near zero when no stimulus was presented. In contrast, VEMPpp and VEMPn values reached a floor above zero that is due to the baseline EMG variation. This figure shows that VEMPid better represents the saccular output than VEMPpp and VEMPn in that when no stimulus is presented the estimated inhibition depth is zero.

To measure how well sound-evoked cVEMPs can be distinguished from no-sound EMG measurements, we used the Cohen's $d$ effect size. Cohen's $d$ indicated that contraction amplitude produced little change in the ability to distinguish cVEMP presence/absence for all 3 cVEMP metrics (Fig. 5). The Cohen's $d$ decreased with age, which can be expected given that older patients have lower outcomes for VEMPpp, VEMPn, and VEMPid (Fig. 2). This indicates that threshold increases with age, which has been shown before [Ochi and Ohashi, 2003; Basta et al., 2017; Singh et al., 2014]. For all the cVEMP metrics, Cohen's $d$ increased with sound level but the differences in Cohen's $d$ across contraction ranges were small for all age groups.

For the no-sound recordings, we performed the same cVEMP computations as were done for sound-on recordings. The values obtained from no-sound recordings are not true cVEMP measurements; instead they show a noise floor for these metrics. The no-sound recordings showed the same trends as the sound-on recordings: VEMPpp increased with muscle contraction amplitude (Fig. 2a-c, diamonds) whereas VEMPn and VEMPid were little influenced by muscle contraction amplitude (Fig. 2d-i, diamonds). This is what is expected considering that as muscle contraction amplitude goes up, r.m.s. EMG goes up and the maximum positive and negative excursions of the EMG go up, which makes VEMPpp go up. In contrast, both VEMPn and VEMPid provide normalization mechanisms that remove the increase due to increasing EMG, so they are little affected by muscle contraction amplitude. 


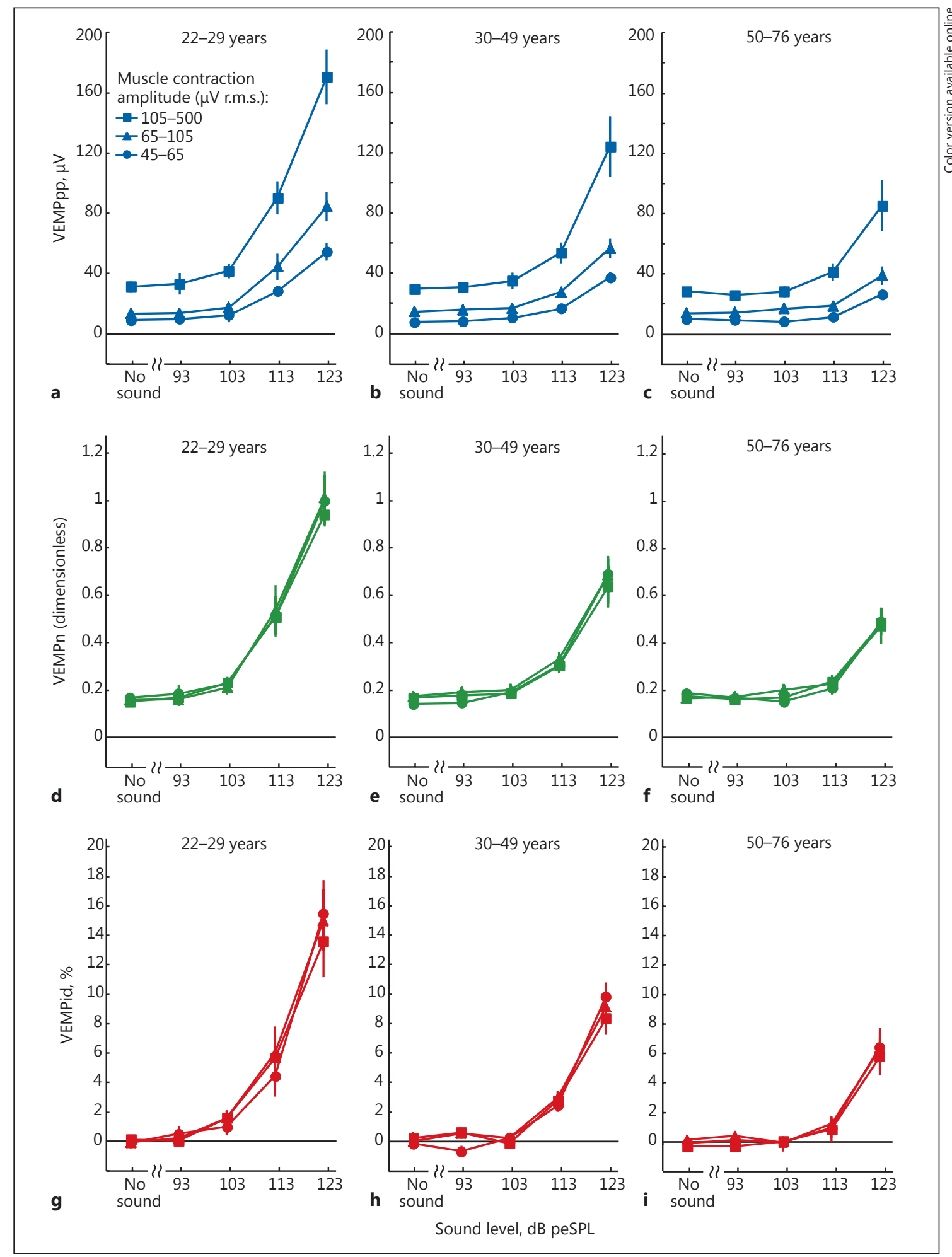

Fig. 4. Average VEMP peak-to-peak (VEMPpp) (a-c), normalized VEMP (VEMPn) (d-f), and VEMP inhibition depth (VEMPid) (g-i) for all age groups as functions of sound level (no sound, 93, 103,113 , and 123 peSPL), showing that in all age groups VEMPpp, VEMPn, and VEMPid all grow with sound level while VEMPid is the only metric that extends down to zero when no sound is presented, suggesting that VEMPid is the most accurate measure of true saccular function. Error bars indicate the standard error of the mean. 


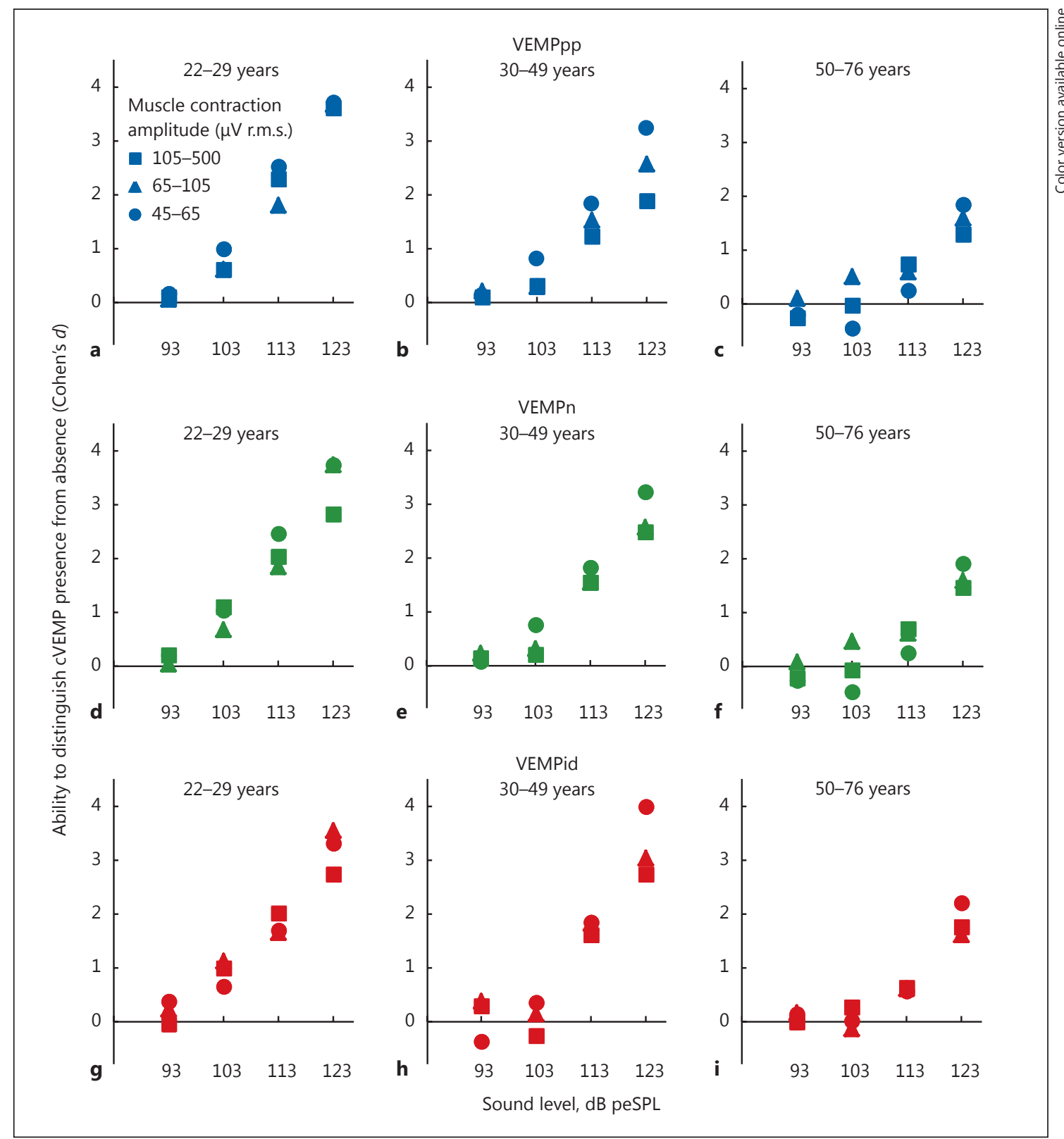

Fig. 5. Cohen's $d$ effect size, which assesses the ability to distinguish cVEMP presence or absence, for VEMPpp (a-c), VEMPn (d-f), and VEMPid ( $\mathbf{g - i})$ for all age groups. Cohen's $d$ increases with an increase in sound level but shows no consistent pattern in the ef- fects of muscle contraction amplitude in any of the age groups, i.e. the contraction amplitudes that produced the highest or lowest Cohen's $d$ vary and seem random.

\section{Discussion}

This study evaluates the effect of muscle contraction amplitude in 25 healthy subjects (50 ears) on $3 \mathrm{cVEMP}$ metrics: raw peak-to-peak amplitude (VEMPpp), normalized peak-to-peak amplitude (VEMPn) and VEMP inhibition depth (VEMPid). Our data show that muscle contraction amplitude strongly affects VEMPpp values but has little effect on VEMPn or VEMPid for 45 to approximately $500 \mu \mathrm{V}$ r.m.s. contraction amplitudes across all sound levels. Although all $3 \mathrm{cVEMP}$ metrics decreased with age, muscle contraction amplitude had little effect on VEMPn and VEMPid in all age groups (Fig. 2). While VEMPn and VEMPid did an equally good job at reducing 
the effects of contraction amplitude, VEMPid is more representative of saccular output because it provides a "meaningful zero": with no stimulus the estimated saccular inhibition is zero (Fig. $4 \mathrm{~g}-\mathrm{i}$ ).

Although we did not ask subjects to target contraction amplitudes below $45 \mu \mathrm{V}$ r.m.s., there are adequate data to show that at most above-threshold sound levels, VEMPn and VEMPid values averaged slightly lower in the 25 - to $45-\mu \mathrm{V}$ r.m.s. range than in the $45-$ to $65-\mu \mathrm{V}$ r.m.s. range. This is consistent with findings by Rosengren [2015], who also found lower normalized peak-to-peak amplitudes at very low contraction levels. Whether contractions below $45 \mu \mathrm{V}$ r.m.s. should be used clinically is uncertain given the reduced amount of available data below $45 \mu \mathrm{V}$ r.m.s. For even lower contraction amplitudes, $<25 \mu \mathrm{V}$ r.m.s., we have no data and cannot say whether such low contraction amplitudes might be usable. Until the use of very low contraction amplitudes is studied more thoroughly we do not recommend using contraction amplitudes $<45 \mu \mathrm{V}$ r.m.s.

We did not explore the upper limit of muscle contraction amplitude and therefore did not determine what upper limit (if any) should be set on muscle contraction amplitudes. Several studies reported that VEMPpps sometimes saturated at very high contraction amplitudes [Colebatch et al., 1994; McCaslin et al., 2014; Rosengren, 2015], with the lowest EMG at which saturation was found being at EMG levels $224 \mu \mathrm{V}$ RECT [Colebatch et al., 1994]. In 1 outlying study, Bogle et al. [2013] found apparent VEMPpp "saturation" starting near $25 \mu \mathrm{V}$ RECT and did not find any EMG range over which VEMPn was constant. The origin of the aberrant findings of Bogle et al. [2013] is unclear but might be from the use of several different head maneuvers to obtain a wide range of contraction amplitudes. The more common finding of nonlinearity only for EMGs $\geq 225 \mu \mathrm{V}$ RECT indicates that very high contraction amplitudes should be avoided. Many patients have trouble contracting their SCM over $65 \mu \mathrm{V}$ r.m.s. (approx.. $50 \mu \mathrm{V}$ RECT) and will never reach amplitudes over $100 \mu \mathrm{V}$ rms with the method of SCM activation used in this study: sitting up straight and turning the head away from the stimulated ear. Nonetheless, some patients can produce high EMG amplitudes. To avoid VEMPpp saturation, they should not be encouraged to contract at extremely high amplitudes.

Prior studies have suggested target muscle contraction amplitudes and explored how well normalization removes the effects of contraction amplitude. Early studies, before normalization became an established technique, considered that to compare VEMPpp measurements across subjects, muscle EMG amplitudes had to be kept constant. Akin et al. [2004] proposed a target level of 30$50 \mu \mathrm{V}$ r.m.s. They did not use normalization, therefore it is unclear whether their data from contractions of 30-50 $\mu \mathrm{V}$ r.m.s. produced the same normalized peak-to-peak amplitude as their data from higher contraction amplitudes. Davenport [2010] proposed a target EMG level of $50 \mu \mathrm{V}$ RECT. McCaslin et al. [2014] looked at how well normalization stabilized the peak-to-peak amplitudes using a design somewhat similar to ours but with high target levels $(100,200,300,400 \mu \mathrm{V}$ RECT) and found normalization produced uniform peak-to-peak amplitudes for contractions 100-300 $\mu \mathrm{V}$ RECT but not for $400 \mu \mathrm{V}$ RECT. Rosengren et al. [2010] recommended 40 to approximately 150-200 $\mu \mathrm{V}$ RECT be used. To minimize the chance of getting high IAR values, in a later study Rosengren [2015] recommended a minimum EMG of $100 \mu \mathrm{V}$ RECT but also suggested that $80 \mu \mathrm{V}$ RECT might be a more reasonable clinical target. Finally, the proposed guidelines for the clinical application of cVEMPs [Papathanasiou et al., 2014] state that "it is desirable that average rectified or RMS muscle activity is kept between 50 and $200 \mu \mathrm{V}$." Here, we show that for contraction amplitudes of $45-300 \mu \mathrm{V}$ r.m.s. (equivalent to $34-227 \mu \mathrm{V}$ RECT), uniform values are produced by normalization of VEMPpp or by using VEMPid. Thus, any contraction within this range should be equivalently useful.

Above we considered the effect of muscle contraction amplitude on the values of cVEMP metrics. However, these values do not fully convey the ability to detect the presence versus absence of a cVEMP response. This ability was evaluated using Cohen's $d$ which indicated that muscle contraction amplitude has little or no effect on the ability to detect cVEMP presence versus absence (Fig. 5). It is notable that VEMPpp does just as well as VEMPn and VEMPid for detecting VEMP presence versus absence. These similar detection abilities can be understood by considering that when visualizing a recording to determine whether a cVEMP is present or not, the waveshape is more important than the amplitude, and normalization changes the amplitude but has little effect on the waveshape. Also, as contraction amplitude goes up, so does the background noise (Fig. 1).

The strengths of our study are that all subjects had to maintain the same predefined muscle contraction amplitudes using a single maneuver - turning the head with different amounts of effort. In addition, muscle contraction was continuously monitored, and contractions outside the targeted range were excluded from analyses of that target range group. Furthermore we tested our subjects at muscle contraction amplitudes that can realistically be 
reached by patients with the method of SCM activation used in this study so that our results will be clinically relevant. In contrast to most other studies, all of our subjects were measured over a range of sound levels, including a "no-sound" control, while evaluating different targeted contraction strengths. This allowed for the analysis of the effects of different contraction amplitudes on different cVEMP metrics at both high and low sound levels. The results showed that normalization works at both low and high sound levels and across age groups. Our results can also be expected to apply to patients, as long as the patient population does not have a muscle control problem. However, this remains to be demonstrated experimentally.

\section{Conclusion}

In summary, although muscle contraction amplitude strongly affects VEMPpp amplitude, throughout the range of 45-500 $\mu \mathrm{V}$ r.m.s. contraction amplitude has little effect on VEMPn or VEMPid, and cVEMP detection is reliable. Clinically, this means there may be no need for subjects to exert maximal or high muscle contraction effort when using VEMPn or VEMPid, which is especially beneficial in patients for whom reaching high levels of SCM contractions can be challenging.

\section{Acknowledgments}

This work was conducted with support from Harvard Catalyst, the Harvard Clinical and Translational Science Center (National Center for Research Resources and the National Center for Advancing Translational Sciences, National Institutes of Health Award UL1 TR001102), and financial contributions from Harvard University and its affiliated academic health care centers.

\section{Disclosure Statement}

There is no conflict of interest.

\section{References}

Akin FW, Murnane OD: Vestibular evoked myogenic potentials: preliminary report. J Am Acad Audiol 2001;12:445-452.

Akin FW, Murnane OD, Panus PC, Caruthers SK Wilkinson AE, Proffitt TM: The influence of voluntary tonic EMG level on the vestibularevoked myogenic potential. J Rehabil Res Dev 2004;41:473-480.

Basta D, Todt I, Ernst A: Characterization of agerelated changes in vestibular evoked myogenic potentials. J Vestib Res 2007;17:93-98.

Bogle JM, Zapala DA, Criter R, Burkard R: The effect of muscle contraction level on the cervical vestibular evoked myogenic potential (cVEMP): usefulness of amplitude normalization. J Am Acad Audiol 2013;24:77-88.

Cohen J: The analysis of variance and covariance. In Cohen J (ed): Statistical Power Analysis for the Behavioral Sciences. New York, Erlbaum Associates, 1988, pp 283-355.

Colebatch JG, Halmagyi GM, Skuse NF: Myogenic potentials generated by a click-evoked vestibulocollic reflex. J Neurol Neurosurg Psychiatry 1994;57:190-197.

Curthoys IS: A critical review of the neurophysiological evidence underlying clinical vestibular testing using sound, vibration and galvanic stimuli. Clin Neurophysiol 2010;121:132144.

Davenport MJ: The Effect of Sternocleidomastoid Muscle Activation Pattern and Feedback Condition on the Vestibular Evoked Myogenic Potential; electronic thesis and disserta- tions, East Tenessee State University, 2010, paper 1776.

Isaacson B, Murphy E, Cohen H: Does the method of sternocleidomastoid muscle activation affect the vestibular evoked myogenic potential response? J Vestib Res 2006;16:187-191.

Lim CL, Clouston P, Sheean G, Yiannikas C: The influence of voluntary EMG activity and click intensity on the vestibular click evoked myogenic potential. Muscle Nerve 1995;18:12101213.

McCaslin DL, Fowler A, Jacobson GP: Amplitude normalization reduces cervical vestibular evoked myogenic potential (cVEMP) amplitude asymmetries in normal subjects: proof of concept. J Am Acad Audiol 2014;25:268-277.

Noij KS, Hermann BS, van Tilburg MJ, Rauch SD, Guinan JJ: Toward optimizing VEMP: calculating VEMP inhibition depth (VEMPid) with a generic template. Assoc Res Otolaryngol Midwinter Meeting 2017, abstract 451, p 40.

Ochi K, Ohashi T: Age-related changes in the vestibular-evoked myogenic potentials. Otolaryngol Head Neck Surg 2003;129:655-659.

Ochi K, Ohashi T, Nishino H: Variance of vestibular-evoked myogenic potentials. Laryngoscope 2001;111:522-527.

Papathanasiou ES, Murofushi T, Akin FW, Colebatch JG: International guidelines for the clinical application of cervical vestibular evoked myogenic potentials: an expert consensus report. Clin Neurophysiol 2014;125:658-666.
Prakash SR, Herrmann BS, Milojcic R, Rauch SD, Guinan JJ Jr: Evaluating inhibition of motoneuron firing from electromyogram data to assess vestibular output using vestibular evoked myogenic potentials. Ear Hear 2015; 36:591-604.

Rauch SD, Zhou G, Kujawa SG, Guinan JJ, Herrmann BS: Vestibular evoked myogenic potentials show altered tuning in patients with Ménière's disease. Otol Neurotol 2004;25:333-338.

Rosengren SM: Effects of muscle contraction on cervical vestibular evoked myogenic potentials in normal subjects. Clin Neurophysiol 2015;126:2198-206.

Rosengren SM, Welgampola MS, Colebatch JG: Vestibular evoked myogenic potentials: past, present and future. Clin Neurophysiol 2010; 121:636-651.

Singh NK, Kashyap RS, Supreetha L, Sahana V: Characterization of age-related changes in sacculocolic response parameters assessed by cervical vestibular evoked myogenic potentials. Eur Arch Otorhinolaryngol 2014;271: 1869-1877.

Van Tilburg MJ, Herrmann BS, Guinan JJ Jr, Rauch SD: Normalization reduces intersubject variability in cervical vestibular evoked myogenic potentials. Otol Neurotol 2014; 35:e222-e227.

Wang CT, Young YH: Comparison of the head elevation versus rotation methods in eliciting vestibular evoked myogenic potentials. Ear Hear 2006;27:376-381. 